\begin{tabular}{|c|c|c|}
\hline Beitr. Ent. & Keltern & ISSN 0005-805X \\
\hline $\mathbf{5 6}(2006) 2$ & S. 422-432 & 15.12 .2006 \\
\hline
\end{tabular}

\title{
Ein dunkelflügliges Weibchen von Calopteryx splendens bei Wesel/Niederrhein mit Diskussion der östlichen ssp. ancilla (SELYS, 1853)
}

Mit 4 Figuren

\author{
EBERHARD G. SCHMIDT
}

\section{Zusammenfassung}

Ein dunkelflügliges o von Calopteryx splendens wurde am 16.07.1995 an der Issel bei Wesel per Fernglas entdeckt und fotografisch dokumentiert (Fig. 1). Es war ein Einzeltier unter Hunderten mit den typisch grünlich hyalinen Flügeln. ㅇ mit ausgedehnt dunklen Flügeln sollen für die osteuropäische ssp. ancilla (Selys, 1853; „race de Prusse“ aus Ostpreußen nach Selys \& Hagen, 1854) typisch sein, jedoch sind aktuelle Daten oder neuere fotografische Belege dazu in der deutschsprachigen Literatur kaum zu finden. In die meisten der gängigen Bestimmungswerke sind dunkelflüglige + , die auf den ersten Blick C. virgo o gleichen, nicht aufgenommen worden. Die ancilla- $\sigma^{\star}$ sind dadurch ausgezeichnet, daß das blaue Band der Flügel proximad weit über den Nodus und distad meistens nahezu oder ganz bis zur Flügelspitze reicht (Fig. 2). Eine Tendenz dazu ist in N-Deutschland (steigend von West nach Ost) zu erkennen (Fig. 3-4). Die Dunkel-Färbung der Flügel bei den wenigen bekannten $q$ ist dagegen recht einheitlich ausgedehnt (entsprechend der Fig. 1). Das spricht genetisch für einen rezessiv-dominanten „Ja-Nein“-Mechanismus, der zugleich die Seltenheit in der Übergangszone erklärte. Es besteht jedoch ein hohes Informationsdefizit zur taxonomischen und geografischen Abgrenzung der Unterart. Es soll mit diesem Beitrag für die dunkelflüglige o -Farbform sensibilisiert und das Monitoring von ancilla vor allem im Gebiet der Oder/Neiße und östlich davon angeregt werden. - Vorangestellt werden eine kritische Diskussion der unscharfen Begriffe „Rasse“ bzw. „andromorph“/,androchrome“ für derartige Farbformen, ein Hinweis auf den Stellenwert der Molekulargenetik für die phylogenetische Systematik und auf die nomenklatorische Problematik der ssp. ancilla im nördlichen Mitteleuropa.

\section{Summary}

A dark-winged female of Calopteryx splendens was observed on 16.07.1995 on the Issel near Wesel through binoculars and recorded photographically (Fig. 1). It was a singleton amongst hundreds with the typical greenish-hyaline wings. Females with extensively dark wings are meant to be typical for the East European subspecies ancilla (Selys, 1853; "race de Prusse" from East Prussia according to Selys \& Hagen, 1854), but recent data or photographic documentation of such specimens are hardly to be found in German language literature. In the majority of current identification works, dark-winged females, which at first glance resemble the male of C. virgo, are not included. The male of ancilla is characterized by the blue band on the wings reaching proximally much further than the nodus and distally mostly nearly or entirely to the wingtip (Fig. 2). A tendency towards this is to be observed in N. Germany (increasing from West to East) (Figs 3-4). Contrarily the dark colour of the wings in the few known females is of quite constant extent (as in Fig. 1). This speaks for a recessive-dominant "Yes-No" mechanism, which also explains the rarity in the transitional zone. There however remains a large deficit on the taxonomic and geographic delimitation of the subspe- 
cies. This contribution is to draw awareness to the dark-winged female form and to encourage monitoring of ancilla, particularly in the Oder / Neiße region and further east. Also included is a critical discussion of the terms "race" and of "andromorph / androchrome" for such colour forms, a remark on the value of molecular genetics for phylogenetic classification and the nomenclatural problems attached to ssp. ancilla in north-central Europe.

Keywords

Odonata, subspecies, colour variety, distribution, sex dimorphism, Eastern Europe

\section{Einf ̈̈h rung}

Bei der Erfassung der Libellenfauna der Issel (rechter Niederhein nahe Wesel; ScHmidt 2004) wurde unter den Massen von Calopterys splendens ein dunkelflügliges beobachtet und fotografiert (Fig. 1). Diese Farbform ist in Deutschland sehr selten und auch nur für das norddeutsche Tiefland angegeben. Sie ist typisch für die aus Königsberg/ Ostpreußen beschriebene „race prussienne (C. ancilla, Hagen)“ bzw. "Race de Prusse" (Selys-Longchamps 1853 bzw. Selys-Longchamps \& Hagen 1854). In aktuellen Feldführern wird eine derartige Farbform kaum erwähnt, ein Hinweis findet sich jedoch u. a. bei Schmidt (1929). Die splendens-Ausbeute der GdO-Exkursion nach NO-Polen 1998 paßt dazu (JöDICKE 1999). Insgesamt ergaben sich bei einer stichprobenhaften Literatur-Recherche verschiedene Unstimmigkeiten. Sie werden nachstehend aufgezeigt und sollen eine Klärung durch gezielte Analysen stimulieren. Eine Diskussion von grundlegenden Begriffen/Methoden wird vorangestellt.

\section{Diskussion der Grundbegriffe "Rasse“ und „homöo-/ androchrome“}

Die Grundeinheit der Systematik ist die Art (species). Sie wird (nach Möglichkeit) als natürliche potentielle Fortpflanzungs-Gemeinschaft definiert und wissenschaftlich mit einem Binomen benannt (Kraus 2000). Genetisch verschiedene, geografisch getrennte Teile einer Art werden als Unterart (subspecies, ssp.) begriffen und mit einem Trinomen benannt (Kraus 2000). Geografisch benachbarte Unterarten sind typisch durch eine Vermischungszone verbunden. Unterarten sind wichtig als Indiz dafür, daß eine Art evolutiv aktiv ist und im Langzeitprozeß einer Artaufspaltung steckt (ScHMIDT 2002). Der deutsche Begriff Rasse bezeichnet dagegen bei Tieren Zuchtformen (Herre \& RöHrs 1990; bei Pflanzen heißen sie Sorten), die im Sinne der wissenschaftlichen Nomenklatur unbeachtliche infrasubspezifische Einheiten und damit zu vernachlässigen sind. Der Begriff Rasse für Menschengruppen dürfte ursprünglich (d. h. vor den großen Migrationen und ethno-kulturellen Normierungen) dem der Unterarten entsprochen haben, er ist also logisch inkonsistent. Vielfach wird der Begriff Rasse gedankenlos zum Anglizismus, wenn das englische „race“ mit Rasse übersetzt wird, denn „race“ umfaßt sowohl die Unterarten („geografische Rassen“) als auch die Rassen im Sinne von Zuchtformen. Der Begriff Rasse sollte daher um der Klarheit willen im wissenschaftlichen Sprachgebrauch nicht anstelle von Unterart benutzt werden (wie bei WiLlmanN 1985, entgegen z. B. Sudhaus \& Rehfeld 1992). 
Bei Libellen sind die reifen + in der Regel anders gefärbt oder gezeichnet als die reifen $\sigma^{*}$, diese Arten sind heterochrom (Ausnahmen z. B. Zwei-/Vierfleck). Es kann jedoch ein Teil der 9 in einzelnen Farb-Merkmalen mit den $\sigma^{*}$ übereinstimmen. Sie werden vielfach als homöochrom oder androchrom bezeichnet. Dieser Begriff erscheint dann zwar als wissenschaftlich, ist aber oft mehrdeutig und damit wertlos. Das Phänomen sollte besser durch eine eindeutige Merkmalsangabe (z. B. „blaue $q$ “ bei Enallagma oder Coenagrion) konkretisiert werden. Analog zu bewerten ist die Bezeichnung „color morphs" (wie bei FINCKE et al. 2005). Überdies bleiben die geschlechtsspezifischen Zeichnungsmuster verschieden. Das gilt auch für die dunkelflügligen + von Calopteryx splendens: Sie haben zwar $\sigma^{\star}$-ähnlich weitgehend opak gefärbte Flügel, jedoch (wie bei dem Beispiel in Fig. 1) mit anderem Muster als bei den $\sigma^{*}$. Sie dürften daher bei Sichtbeobachtung eher mit virgo- $\sigma^{*}$ verwechselt als splendens zugeordnet werden (vgl. SteIneCke 1940). Allerdings weichen sie von den $\sigma^{*}$ durch ein relativ großes weißes Pseudo-Pterostigma und die Grünfärbung (statt Blau) des Körpers ab. Ganz unsinnig ist die Bezeichnung „,andromorph“, da die geschlechtsspezifischen Gestalts-Unterschiede nicht verändert werden. Das gleiche gilt für das Gegenstück „gynomorph“ (bei McKeE et al 2005 für von den $\sigma^{\star}$ abweichende Grundfärbung der 우, bei Coenagrion puella z. B. vornehmlich grün [„brown/olive“]; die + mit grünem Thorax und blauer Grundfarbe der Abdomen-Oberseite bleiben unberücksichtigt!).

\section{Eine Anmerkung zum Stellenwert molekularbiolo- gischer Taxonomie}

Aktuell wird vielfach die Analyse von Gen-Sequenzen als Königsweg der phylogenetischen Systematik angesehen. Es handelt sich jedoch um eine numerische Unterschiedsanalyse, also um numerische Taxonomie. Diese ist mit der phylogenetischen Systematik prinzipiell nicht kompatibel, denn bei dieser steht die Bewertung der Merkmale nach ursprünglich (plesiomorph) und abgeleitet (apomorph) im Mittelpunkt, nur (gemeinsame) abgeleitete (also innovative) Merkmale (Synapomorphien) begründen eine phylogenetische Verwandtschaft. Molekularbiologische Stammbäume repräsentieren daher nur ein künstliches, nicht ein natürliches System. Sie können daher auch nicht die Abgrenzung höherer Taxa (wie Gattungen: „Erythromma“ lindenii), die sowieso nur abstrakte Denkkategorien darstellen, objektivieren. Oft werden sie auch noch mit Zeitangaben für die Gabelstellen versehen. Voraussetzung dafür wäre (u. a.) eine konstante Veränderungsrate, das ist aber eine irreale Annahme! Die Art ist dagegen (im Gegensatz z. B. zu Gattungen) eine reale Kategorie (potentielle natürliche Fortpflanzungs-Gemeinschaft). Sie kann ebenso wenig nach molekularbiologischen wie nach morphologischen Unterschieden abgegrenzt werden, denn nach dem gängigen „biologischen Artkonzept“ ist nach einer Fortpflanzungsschranke unter natürlichen Bedingungen zu fragen, es kann also nur im Überlappungsbereich der Areale eine Antwort geben. Bei Unterarten wird durch molekulare Genanalysen das Spektrum der Merkmale, die eine Unterbrechung des Genflusses und divergierende Entwicklung belegen und damit den Status als ssp. verifizieren, erweitert. Hier sind sie daher sehr hilfreich, benötigen aber wie die klassischen Untersuchungen größere Stichproben und (wie beim Beispiel Calopteryx splendens mit einfach erkennbaren Farbmerkmalen) einen deutlich höheren Aufwand (zur Theorie der phylogenetischen Systematik vgl. z. B. Ax 1984, Hennig 1969, Mayr 1969, Sudhaus \& Rehfeld 1992, Willmann 1985). 
Die Definition der ssp. ancilla Selys, 1853 , nach der Flügelfärbung

Calopteryx splendens (Harris, 1782) wurde aus England (locus typicus) beschrieben (kommentierter Text bei LuCAS 1900: $221 \mathrm{ff}$.). Die Nominat-Unterart splendens splendens (Harris, 1782) ist dort relativ einheitlich gefärbt. Der dunkle Flügelfleck der $\sigma^{\text {t }}$ bildet ein rundliches bis rechteckiges Band, es beginnt typisch am Nodus (gerade abgeschnitten oder leicht konvex zur Basis hin) und endet etwa dort, wo sich der Vorderrand zur Spitze hin krümmt. An der Flügel-Spitze ist damit ein etwa halbkreis-/halbmond-förmiger Teil hyalin (typisch auf Tf. 13 in Hammond \& Merritt 1983, S. 57). Die + haben stets grünlich-hyaline Flügel. - Die Unterart splendens ancilla Selys, 1853 (locus typicus Königsberg, das jetzt russische Kaliningrad, im früheren Ostpreußen) ist durch die proximale Ausdehnung des dunklen Flügelflecks bei den $\sigma^{\text {t }}$ definiert worden (SelysLongchamps \& Hagen 1954): Er ist zur Flügelbasis hin konvex und reicht bis in die Nähe des Flügel-Vierecks, also über mehr als die Hälfte des Raumes zwischen FlügelBasis und Nodus. Am Flügel-Vorderrand beginnt er zumindest einige (meistens über 10) Antenodal-Queradern proximat vom Nodus. Außerdem ist der dunkle Flügelfleck auch zur Spitze hin ausgedehnt, typisch bleibt nur ein schmaler, sichelförmiger Saum hyalin, der Fleck kann auch die Spitze voll einnehmen (Fig. 2; zur ähnlichen, im Balkan benachbarten ssp. balcanica Fudakowski, 1930, vgl. FudAKowski 1930 und ADAmovic \& Vijatov 1996). Die Formen mit dunkler Flügelspitze (Fig. 2) hat Scholz (1908) aus Schlesien als var. Tümpeli bezeichnet, hier ohne Bezug zum Geschlecht, (1917) dagegen

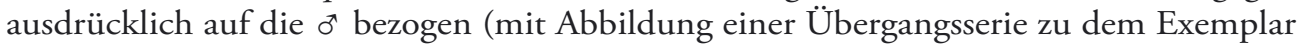
von 1908). Einen Vergleich mit splendens ancilla Selys, 1853, hat ScHolz unterlassen, die Nennung von ancilla für Polen (Scholz 1917, S. 93) zitiert nur kommentarlos einen anderen Sammler.

Bei den 9 ist im Vergleich mit der Nominatform generell das weiße Pseudopterostigma vergrößert, es reicht über 6-8 Zellen. ㅇ mit dunklen Flügeln kommen (im Gegensatz zur Nominatform) vor (hinreichendes, aber nicht notwendiges Merkmal der Unterart). Die Flügel scheinen recht einheitlich auf voller Breite dunkel von der Flügelspitze (höchstens ein schmaler hyaliner Saum) bis in die Nähe vom Flügelviereck gefärbt zu sein. Diese dunkelflügligen + ähneln daher bei Sichtbestimmung auf den ersten Blick virgo $\sigma^{\star}$, doch ist der Körper grün, nicht blau und das weiße Pseudopterostigma schließt Verwechslungen mit $\sigma^{\text {t }}$ aus.

Hinzuweisen ist darauf, daß ancilla in systematischen Übersichten oft unzureichend oder fehlerhaft berücksichtigt wird. So zitiert sie Kirby (1890) als „Var. a. Calopt. Ancilla" [Selys 1853], das gleiche gilt für JöDicke (1999), dabei steht im Original unter Calopteryx splendens Harris (S. 13) „Race prussienne (C. ancilla, Hagen)“. DAvis \& ToBIN (1984) führen ancilla als eigene Art, bei TsudA (1991) ist ancilla in die Synonymie von C. splendens mingrelica (ostmediterran!) eingereiht. Ancilla Hagen ist ein irrelevanter Manuskript-Name, es zählt die Publikation durch Selys (1853).

Im östlichen Mitteleuropa wird der Name ancilla vielfach als nomenklatorisch irrelevante Farbvariante („var.") für dunkelfüglige splendens- 9 aufgefaßt, so bei FuDAKOwsKi (1932) für Polen und Spuris (1956) für Lettland (vgl. auch JöDicke 1999). Das widerspricht aber der ursprünglichen Definition von ancilla Selys, 1853 und der taxonomischen Situation. Dabei sind dunkelflüglige + nach dem derzeitigen 
Kenntnisstand in Osteuropa eine hinreichende Bedingung für die Zuordnung zur ssp. splendens ancilla.

Entgegen LoHMANN (1992a,b) werden hier westmediterrane splendens-Formen mit Flügel-Fleck (fast) bis zur Spitze (wie caprai Conci \& Nielsen, 1956) nicht mit ancilla synonymisiert (auch entgegen Aguilar \& Dommenget 1998; die dortige Fig. Tf.1, fig. 3 a sub ancilla ist caprai!) und nicht in die Diskussion einbezogen! Überlegungen von LoHmann (1992a,b) zu eiszeitlichen Refugien und nacheiszeitlichen Ausbreitungswegen (im Sinne von Reinig 1938, LatTin 1967) werden als zu spekulativ angesehen und nicht weiter verfolgt.

\section{Nomenklatorische Pikanterien}

Selys-Longchamps hat die Unterarten von Calopteryx splendens primär (nomenklatorisch irrelevant) nach dem Areal bezeichnet, den Namen ancilla (mit nomenklatorisch irrelevanter Zuordnung zu HageN) bei der Kurzbeschreibung 1853 auch nur in Klammern gesetzt, bei der ausführlichen Charakteristik 1854 gar nicht beigefügt (s. o.). Es fehlt jedoch jeder Hinweis auf die Beschreibung von Calopteryx splendens unter dem Namen Agrion parthenias bei Charpentier (1840; locus typicus Schlesien). Die dortige Abbildung zeigt beim $\sigma^{*}$ eine breite dunkle Flügelbinde, die fast bis an die Spitze reicht, doch wird an der Basis der Nodus proximat nur wenig überschritten, der Rand ist fast gerade (nicht deutlich konvex: Fig. 2). Das entspricht den Tieren aus NO-Polen bei JöDICKe (1999). Das ebenfalls abgebildete $q$ hat hyaline Flügel und hilft hier nicht weiter. Es wird aber das gelegentliche Vorkommen von dunkelflügligen + im Text erwähnt. Parthenias Charpentier entspricht so zumindest dem Übergangsbereich von ancilla zu splendens splendens. Nomenklatorisch ist der Name parthenias nicht Charpentier zuzuordnen, Priorität als Autor hat vielmehr Burmeister 1839. Er hatte zwar das Manuskript von Charpentier berücksichtigen können, schrieb dem entsprechend auch Charpentier die Autorenschaft zu, doch erschien sein Buch ein Jahr früher als das von Charpentier, und Manuskript-Namen gelten nomenklatorisch nicht (Kraus 2000; zu entsprechenden Problemen mit der Autorenschaft bei den Gattungsnamen Sympecma bzw. Platycnemis vgl. Longfield 1954). Burmeister (1839) hatte (anders als Charpentier 1840) nur die splendens splendens Merkmale aufgenommen, nicht die von splendens ancilla; dennoch ist parthenias Charpentier, 1840, als jüngeres Homonym zu parthenias Burmeister, 1839, auch für den Rang der Unterart nicht mehr verfügbar. Damit kann splendens parthenias Charpentier, 1840, nicht das jüngere Synonym ancilla Selys, 1853, ersetzen. Die ancilla-Merkmale sind übrigens auch schon (unter bezug auf Hagen für Ostpreußen) bei Selys-Longchamps \& Hagen 1850 als Ausnahme angeführt, jedoch ohne Vergabe eines eigenen Namens. In seiner Dissertation (1840) stellt HAGEN zwar parthenias Burmeister, 1839, und parthenias Charpentier, 1840, in die Synonymie von splendens (bei ihm unter dem Namen Callepteryx loudoviciana Leach), er geht aber auf die ancilla-Farbformen nicht ein. Das entsprechende Material hat er wohl erst in den 40ern gefunden. 
Zur Verbreitung von ancilla in Mitteleuropa und darüber hinaus

Die Information zum Vorkommen dunkelfügliger splendens- o (im Sinne der ssp. ancilla) im heutigen Deutschland sind äußerst dürftig. In den gängigen Bestimmungsbüchern (wie Bellmann 1993, Jurzitza 2000, Lehmann \& Nüss 1998; auch bei Askew 1988) fehlt jeder Hinweis (vgl. dagegen z. B. Schiemenz 1953, Schmidt 1929, Stresemann \& Klausnitzer 2005, Tümpel 1901). Auch bei Rüppell (et al. 2005) wird auf ancilla de facto nicht eingegangen, die dortigen Calopteryx-Fotos enthalten überdies keine Ortsangaben (anders als z. B. bei Bellmann 1993, Sternberg \& Buchwald 1999) und sind daher zu Unterarten nicht verwertbar. - Bekannt ist mir ein Foto, das möglicherweise aus der Lüneburger Heide stammt (Martens 1982, S. 65), wo ClausNitZer (mdl. Mitt.) die Form einmal gesehen hat. Für Stettin liegt eine alte Meldung vor (KRÜGER 1925). In Ostpolen dürften dunkelflüglige- 9 (wie im früheren Ostpreußen: SelysLongChamps 1853, Selys-Longchamps \& Hagen 1854) regelmäßig neben den + mit grünlich hyalinen Flügeln vorkommen (FUDAKOwski 1930, 1932, JöDicke 1999). Die Informationslücke vor allem im Gebiet von Oder und Weichsel ist dringend zu schließen.

Bei den $0^{*}$ kommen Tiere mit einem dunklen Fleck, der \pm bis in die Flügelspitze reicht (Fig. 3/4), nach eigenen Erfahrungen vereinzelt auch in Westfalen und (weniger) in Schleswig-Holstein vor; in Mecklenburg (Warnow-Exkursion auf der SIOTagung Schwerin 2004), erst recht in Ostbrandenburg (Märkische Schweiz bei Buckow: Sammlung im Deutschen Entomologischen Institut in Müncheberg) sind sie (nach den beiden kleinen Stichproben) häufiger. Scholz (1917) nennt einen Gradienten für die Verdunkelung der Flügelspitze von $10 \%$ an der (damals schlesischen) Oder auf $60 \%$ an der Narew in Ostpolen. Seiner var. Tümpeli Scholz, 1908, entspricht ein Foto des Naturfotografen EBERLE, aufgenommen in Ostpreußen (Fig. 2; abgedruckt z. B. in Steinecke 1940, Naumann 1956). Damit zeichnet sich ein Gradient von West nach Ost für das Gebiet der Norddeutsch/polnischen Tiefebene ab. Auch die Ausdehnung proximat an der Flügelbasis kann in Norddeutschland ancilla entsprechen, allerdings wird am Flügelvorderrand (Costalfeld) der Nodus in den mir bekannten Beispielen kaum überschritten. - Die Funde von dunkelflügligen 9 sind dagegen in Norddeutschland äußerst sporadisch, aber anscheinend uniform in der Flügelfärbung. Das spricht für eine rezessive „Ja-Nein“-Vererbung des Merkmals bei den + (vgl. dazu Mertens et al. 1992, S. 444).

Ancilla ist offenbar eine osteuropäische Form (Arealkarte bei LoHmann 1992a, vgl. LoHMAnN 1992b; berechtigte Kritik bei JöDicke 1999; das ơ-Foto aus Finnland bei KarJalainen (2002) zeigt auch eine typische splendens splendens statt ancilla; zur Variationsbreite in Finnland vgl. VALle 1927). Für Lettland führt SpUris (1956) ancilla (als Name für dunkelflüglige o) neben splendens splendens an. Auch hier ist die Datenlage dürftig. Das norddeutsche Tiefland wäre dann das Übergangsgebiet.

Zum Vorkommen derartiger $0^{7}$-Formen oder von dunkelfügligen ㅇ im südlichen Deutschland (Sachsen: Brockhaus \& Fischer 2005; Thüringen: Zimmermann et al. 2005; Bayern: Kuhn \& Burbach 1998; Baden-Württemberg; Sternderg \& Buchwald 1999) liegen keine Angaben vor. Es ist daher auch bei den ơ eine Informationslücke zu beklagen. Foto-Belege können hier naturschutzkonform helfen. 


\section{Fa z i t}

Calopteryx splendens ancilla Selys, 1853 dürfte als eine gute Unterart für das Gebiet von Osteuropa anzusehen sein. Ihr Vorkommen und der Übergang zu splendens splendens sind jedoch in Mitteleuropa bislang nur unzureichend untersucht. Es wird hiermit angeregt, verstärkt Daten zu sammeln. Passende Fotobelege sind dafür geeignet und lassen sich naturschutzkonform erlangen. Dabei ist bei den $\sigma^{*}$ gezielt nach Extrema (größte/ kleinste Ausdehnung) zu suchen, der Mittelwert abzuschätzen. Der Nachweis von dunkelflügligen- $q$ hat einen besonderen Stellenwert.

\section{Danksagung}

Herzlich Dank sagen möchte ich dem Kollegen BostjaAn Kiauta (Bilthoven/Holland) für eine Kopie des Textes zu ancilla aus Selys 1853.

\section{Literatur}

Adamovic, Z. \& Vijatov, S. 1996: Morphometric Examination of Calopteryx balcanica Fudakowski, 1930, and C. splendens ancilla Selys, 1853 (Zygoptera: Calopterygidae). - Odonatologica 25 (2): 109-118.

Aguilar, J. D' \& Dommanget, J. 1998: Guide des Libellules d'Europe et d'Afrique du Nord. 'identification et la biologie de toutes les espèces. - Delachaux \& Niestlé, 2. Aufl., Lausanne/Paris.

Askew, R. 1988: The Dragonflies of Europe. - Harley, Great Horkesley (2. Aufl. 2004).

Ax, P. 1984 : Das Phylogenetische System. Systematisierung der lebenden Natur aufgrund ihrer Phylogenese. - Fischer Verl., Stuttgart.

Bellmann, H. 1993: Libellen beobachten, bestimmen. - Naturbuch/Weltbild, Augsburg (verändert gegenüber der gleichnamigen Ausgabe von 1987 bei Neumann/Neudamm, Melsungen).

Brockhaus, T \& Fischer, U. (Hrsg.): Die Libellenfauna Sachsens. - Natur \& Text, Rangsdorf.

Burmeister, H. 1839: Handbuch der Entomologie. - Bd. 2, Besondere Entomologie, 2. Abt. Kaukerfe/ Gymnognatha, (erste Hälfte, vulgo Orthoptera). Enslin, Berlin [1838].

Charpentier, T. De 1840: Libellulinae Europaeae descriptae ac depictae. - Leipzig, Voss.

Conci, C. \& Nielsen, C. 1956: Fauna d'Italia: Odonata. - Calderini, Bologna.

Davies, A. \& Tobin, P. 1984: The Dragonflies of the World: A systematic list of extant species of Odonata. Vol. 1: Zygoptera, Anisozygoptera. - Societas Internationalis Odonatologica [SIO]. Rapid Communications (Supplements) No. 3. Utrecht

Fincke, O.; Jödicke, R.; Paulson, D. \& Schultz, T. 2005: The evolution and frequency of female color morphs in Holarctic Odonata: why are male like females typically the minority? - International Journal of Odonatology, Leiden 8 (2): 183-212.

Fudakowski, J. 1930: Über die Formen von Calopteryx splendens Harr. aus Dalmatien und Herzogovina. (Odonata). - Annales Musei Zoologici Polonici, Warszawa 9 (6): 57-63, Tf. 10.

Fudakowski, J. 1932: Neue Beiträge zur Odonaten-Fauna Polens. - Fragmenta Faunistica Musei Zoologici Polonici, Warszawa 1 (15): 389-411.

Hagen, H. 1840: Synonymia Libellularum Europaearum. Dissertatio Inauguralis. - Medizinische Fakultät der Universität Königsberg (Regimonti Prussorum). Dalkowski, Königsberg.

Hammond, C. \& Merritt, R. 1983: The Dragonflies of Great Britain and Ireland. - 2. Aufl., Harley, Great Horkesley.

Hennig, W. 1969: Die Stammesgeschichte der Insekten. - Kramer, Frankfurt/M.

Herre, W. \& Röhrs, M. 1990: Haustiere - zoologisch gesehen. - 2. Aufl., Fischer Verl., Stuttgart.

JöDicke, R. 1999: Libellenbeobachtungen in Podlasie, Nordost-Polen. - Libellula 18 (1/2): 31-48. 
Jurzitza, G. 2000: Der Kosmos-Libellenführer. Die Arten Mittel- und Südeuropas. - 2. Aufl., Franckh/ Kosmos, Stuttgart.

Karjalainen, S. 2002: „Die Libellen Finnlands“ (finnisch). Tammi, Helsinki.

Kirby, W. 1890: A Synonymic Catalogue of Neuroptera Odonata or Dragonflies. With an Appendix of Fossil Species. - Gurney \& Jackson, London, \& Friedländer, Berlin.

Kraus, O. 2000: Internationale Regeln für die Zoologische Nomenklatur. Angenommen von der Int. Union of Biol. Sciences. Offizieller deutscher Text. 4. Aufl. - Goecke \& Evers, Keltern (f. d. Naturwiss. Verein Hamburg).

KRÜGER, L. 1925: Die Odonaten oder Libellen. Eine Einführung in das Studium der Libellen mit einer Übersicht der pommerschen Fauna. - Abhandlungen und Berichte der Pommerschen Naturforschenden Gesellschaft, Stettin 6: 53-106.

Kunn, K. \& Burbach, K. (Bearbeiter, 1998): Libellen in Bayern. - Ulmer Verl., Stuttgart.

LatTin, G. DE 1967: Grundriß der Zoogeographie. - Fischer Verl., Jena.

Lehmann, A. \& Nüss, J. 1998: Libellen. Bestimmung, Verbreitung, Lebensräume und Gefährdung aller Arten Nord- und Mitteleuropas sowie Frankreichs unter besonderer Berücksichtigung Deutschlands und der Schweiz. - 5. Aufl., DJN (Deutscher Jugendbund für Naturbeobachtung), Hamburg.

Lohmann, H. 1992a: Amphiadriatic Faunal Elements in the Genera Calopteryx LeaCH and Cordulegaster LEACH in Southern Italy (Zygoptera: Calopterygidae; Anisoptera: Cordulegastridae). - Notulae Odonatologia, Utrecht 3 (9): 152-153.

Lohmann, H. 1992b: Ein Beitrag zum Status von Coenagrion freyi (BıLek, 1954) und zur subspezifischen Differenzierung von C. hylas (Trybom, 1889), C. johanssoni (Wallengren, 1894) und C. glaciale (SelYs, 1872), mit Bemerkungen zur postglazialen Ausbreitung ostpaläarktischer Libellen (Zygoptera: Coenagrionidae). - Odonatologica 21 (4): 421-442.

Longfield, C. 1954: Nomenclature of the European Species of Odonata (Dragonflies). - The Entomologist's Monthly Magazine, Third Series, London 90: 145-148.

LuCAS, W. 1900: British Dragonflies (Odonata). - Upcott Gill, London.

McKee, D.; Harvey, I.; Thompson, D. \& Sherrat, T. 2005: Frequency of Female Colour Morphs in Populations of four Coenagrionid Damselflies (Zygoptera: Coenagrionidae). - Odonatologica 34 (1): 37-49.

Martens, J. 1982: Heidebäche und -flüsse. Die Perlenfischer von der Ilmenau. S. 62-71 in U. Klugmann (Red.): Naturmagazin draußen: Lüneburger Heide. HB, Hamburg.

MaYr, E. 1969: Principles of Systematic Zoology. - McGraw-Hill, New York.

Mertens, J.; Coster, W.; Meyer, H. \& Dumont, H. 1992: A Method for the Quantitative Analysis of Wing Spots applied to two Populations of Calopteryx splendens (Harris) (Zygoptera: Calopterygidae). Odonatologica 21 (4): 443-451.

Naumann, H. 1952: Wasserjungfern oder Libellen. - Die Neue Brehmbücherei 55. Ziemsen, Wittenberg.

ReINIG, W. 1938: Elimination und Selektion. Eine Untersuchung über Merkmalsprogressionen bei Tieren und Pflanzen auf genetisch- und historisch-chorologischer Grundlage. - Fischer Verl., Jena.

Rüppell, G.; Hilfert-Rüppell, D.; Rehfeldt, G. \& Schütte, C. 2005: Die Prachtlibellen Europas. - Die Neue Brehmbücherei 654. Westarp. Wiss., Hohenwarsleben.

Schiemenz, H. 1953: Die Libellen unserer Heimat. - Urania Verl., Jena.

Sснмidт, Ев. 2002: Studien zur Evolution vor Ort: Das Thema „Art und Artbildung“ anschaulich am Beispiel der Zebras in Zoo und Museum. - Tier und Museum (Bonn) 8 (1/2): 22-36.

Sснмірт, Ев. 2004: Die Auswirkung von Stauhaltungen auf die Libellen-Fauna eines ausgebauten Flachlandbaches, der Issel bei Wesel/Niederrhein (Odonata: Calopterygidae). - Entomologia Generalis 27 (2): 87-104.

Schмidt, Er. 1929: 7. Ordnung: Libellen, Odonata. (Schillebolde, Teufelsnadeln, Augenstecher, Wasserjungfern). - In: „Die Tierwelt Europas“ (Hrsg. Brohmer, P.; Ehrmann, P. \& Ulmer, G.), Bd. 4, Lief. 1 b (66 S.). Quelle \& Meyer, Leipzig. 
Scholz, E. 1908: Die schlesischen Odonaten. - Zeitschrift für wissenschaftliche Insektenbiologie, Husum 4 (11): 417-420, (12): 457-462.

Scholz, E. 1917: Beitrag zur Kenntnis der Odonaten Polens. - Zeitschrift für wissenschaftliche Insektenbiologie, Husum 13: 85-96.

Selys-Longchamps, E. de \& Hagen, H. 1850: Revue des Odonates ou Libellules d'Europe. - Muquardt, Brüssel \& Leipzig; Roret, Paris.

Selys-Longchamps, E. De 1853: Synopsis des Calopterygines. - Bulletins de l'Académie Royale des Sciences, des Lettres et des Beaux-Arts de Belgique, Bruxelles 20 (Annexe): 1-73.

Selys-Longchamps, E. De [\& H. Hagen] 1854: Monographie des Calopterygines. - Muquardt, Brüssel \& Leipzig; Roret, Paris.

Spuris, Z. 1956: „Die Libellen der lettischen SSR“ (russisch). - Akademiya Nauk Latviyskoy SSR, Riga.

Steinecke, F. 1940: Der Süßwassersee. Die Lebensgemeinschaft des nährstoffreichen Binnensees. - Quelle \& Meyer, Leipzig.

Sternberg, K. \& Buchwald, R. (Hrsg. 1999): Die Libellen Baden-Württembergs. Bd. 1: Allgemeiner Teil, Kleinlibellen (Zygoptera). - Ulmer Verl., Stuttgart.

Stresemann, E. (Begründer); Klausnitzer, B. (Herausgeber): Exkursionsfauna von Deutschland Bd. 2, Wirbellose: Insekten. 10. Aufl., Elsevier/Spektrum Verl., Heidelberg.

Sudhaus, W. \& Rehfeld, K. 1992: Einführung in die Phylogenetik und Systematik. - Fischer Verl., Stuttgart.

Tsuda, S. 1991: A Distributional List of World Odonata 1991. - (Selbstverlag?) Osaka, 1991.

TüMpEL, R. 1901: Die Geradflügler Mitteleuropas. - Wilkens, Eisenach.

VALLE, K. 1927: Zur Kenntnis der Odonatenfauna Finnlands III. Ergänzungen und Zusätze. - Acta Societatis pro Fauna et Flora Fennica, Helsingfors 56 (11): 1-36, Karte.

Willmann, R. 1985: Die Art in Raum und Zeit. Das Artkonzept in der Biologie und Paläontologie. - Parey Verl., Hamburg.

Zimmermann, W.; Petzold, F. \& Fritzlar, F. 2005: Verbreitungsatlas der Libellen (Odonata) im Freistaat Thüringen. - Thüringer Landesanstalt für Umwelt \& Geologie (Naturreport 22), Jena.

\section{Anschrift des Verfassers:}

Prof. em. Dr. Eberhard G. Schmidt

Coesfelder Strasse 230

D - 48249 Dülmen

(D: Biologie/-didaktik, S05, Univ. Duisburg-Essen, 45117 Essen).

\section{Subject editor:}

E. K. Groll 
Beitr. Ent. 56 (2006) 2
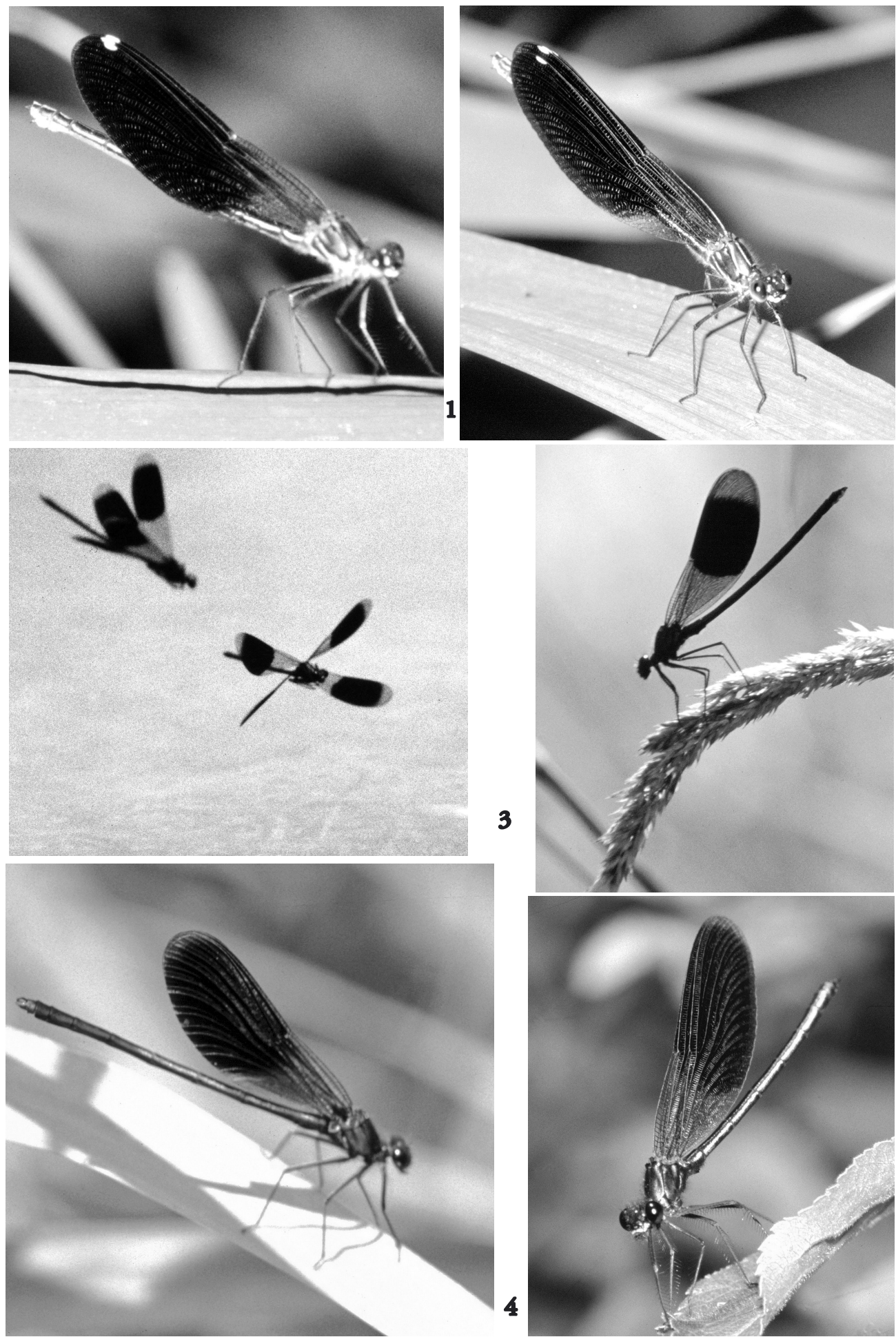

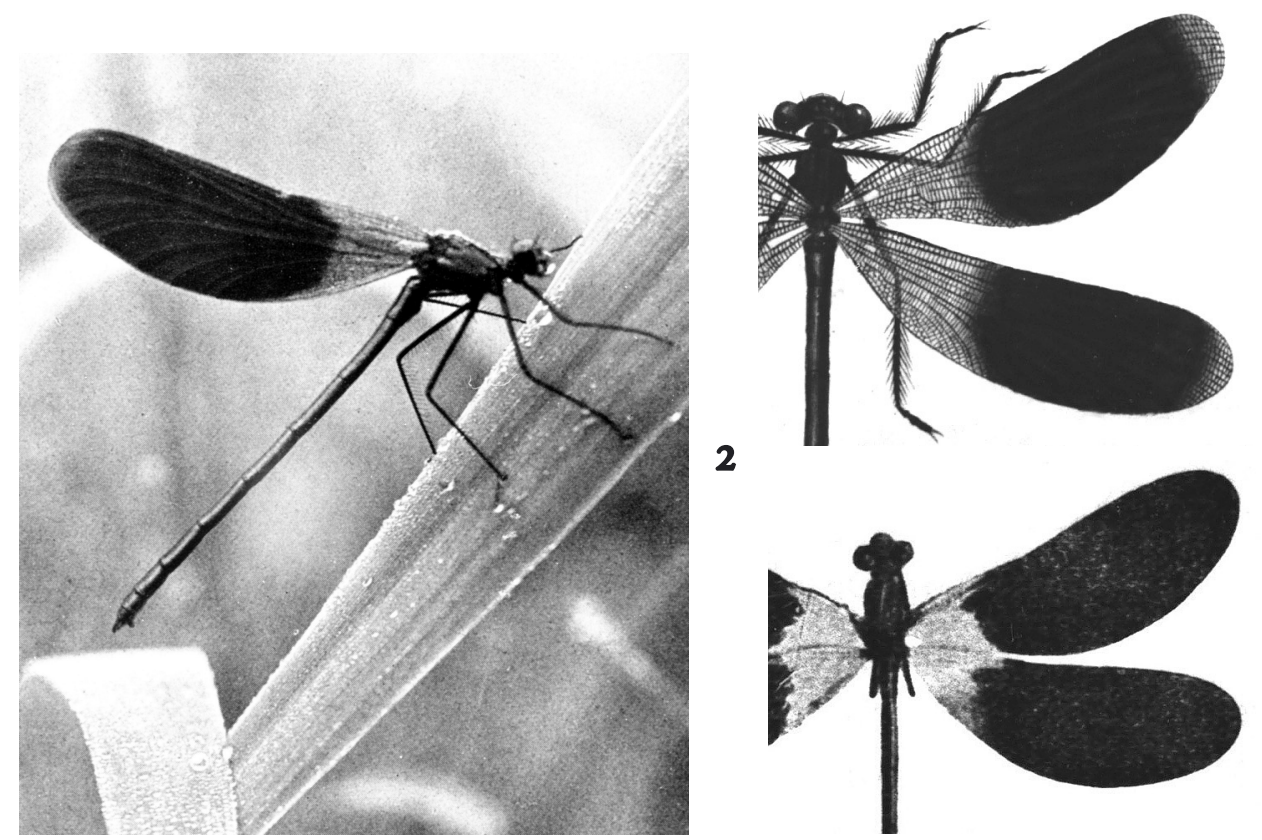

Fig. 2: Reproduktionen zu Calopteryx splendens ancilla. Links ơ mit eindeutiger ancilla-Flügel-Schwärzung (Foto G. Eberle, aus STeinecke, 1940, Fig. 77/S. 125, hier fälschlich als C. virgo bezeichnet; auch in Naumann, 1952, Bild 1/S.6, als „Östliche Rasse“ bezeichnet) aus Ostpreußen, also aus dem Gebiet des locus typicus von ancilla. - Rechts oben rechte Flügel des ơ von Agrion parthenias aus CHARPENTIER (1840; Tafel 33, Ausschnitt). - Rechts unten rechte Flügel der Var. Tümpeli aus Scholz (1908, S. 461, Ausschnitt).

\section{Legenden zu den Figuren von Seite 431.}

Fig. 1: Ein dunkelflügliges 우 von Calopteryx splendens vom Niederrhein (Issel bei Wesel, 26.07.1995).

Fig. 3: $0^{\top}$ von Calopteryx splendens. Links: Beim Drohflug wird die artspezifische Flügelzeichnung vorgewiesen; NO-Ungarn (b. Oros), 17.06.1986: Der dunkle Flügelfleck ist etwas zur Flügelspitze hin vergrößert. - Rechts: $\sigma^{\star}$ auf der Warte am Bachrand (Issel, wie Fig. 1, 18.07.1999); der Flügelfleck entspricht der Nominatform.

Fig. 4: $\sigma^{\top}$ von Calopteryx splendens mit vergrößertem Flügelfleck, an der Flügelspitze ist ähnlich C. splendens ancilla nur ein hyaliner Saum vorhanden. Links Issel (wie Fig. 1, 2 rechts), 18.07.1999; rechts Warnow/ Mecklenburg (Eickhof), 28.07.2004. 